\title{
Active control of the optical properties of nanoscale coatings using 'smart' nanoparticles
}

\author{
Michael B. Cortie, Michael Barnett and Michael J. Ford
}

Institute for Nanoscale Technology, University of Technology Sydney, PO Box 123, Broadway, NSW 2007, Australia

Phone +61-2-9514-2208

Fax +61-2-9514-8349

Email michael.cortie@uts.edu.au

\begin{abstract}
Coatings that can self-modulate their optical properties as a function of an external stimulus are of significant technological interest. In this regard, the possibilities for thermo- or electrochromic materials such as $\mathrm{VO}_{2}$ and $\mathrm{WO}_{3}$ are already comparatively well-known. Here, however, we explore a new kind of 'smart' coating, based on the active control of a plasmon resonance in nanoparticles. One possible system is based on the modulation of the plasmon resonance of a precious metal nanorod or nanosphere by an active dielectric shell. The active dielectric undergoes an insulator-to-metal transition on increase of temperature which modulates the plasmon resonance of the underlying precious metal nanoparticle, thereby changing the wavelength at which its optical extinction is maximum. In the case of nanorods, the absorption maximum of the longitudinal plasmon is particularly sensitive to the aspect ratio of the nanoparticle and the dielectric properties of the environment, and may be readily tuned across the visible and near-infrared portions of the spectrum. In addition, nanoparticles of certain thermochromic dielectrics, such as $\mathrm{VO}_{2}$, are expected to have a plasmon resonance of their own which can be switched on or off by control of the temperature. We consider some of the possibilities, using both the discrete dipole approximation and the exact analytical solution due to Mie to calculate the optical properties.
\end{abstract}

Keywords : nanorods, gold, silver, plasmon resonance, thermochromic, vanadium dioxide, active plasmonic control

\section{INTRODUCTION}

Nanoparticles of certain compounds and elements undergo a plasmon resonance with light. This phenomenon has been particularly well studied in the coinage metals $(\mathrm{Cu}, \mathrm{Ag}, \mathrm{Au})$ for at least two reasons : (i) these nanoparticles are relatively stable under ambient conditions and, (ii) their resonances occur in, or near to, the visible part of the spectrum ${ }^{1-}$ 3. The single dipole plasmon resonance of spherical nanoparticles splits into a transverse and a longitudinal mode as the particle becomes elongated into rod-like shapes. The absorption maximum of the longitudinal plasmon is particularly sensitive to the aspect ratio of the nanoparticle, and may be readily tuned between 450 and $700 \mathrm{~nm}$ for $\mathrm{Ag}$, or 550 to $1500 \mathrm{~nm}$ for Au. There has been some interest in using such precious-metal particles for chemical sensing, e.g. ${ }^{4}$ and medical applications, e.g. ${ }^{5,6}$, however these are not the topics that we will address here. Rather, the present paper is focused on the exploitation of the optical properties of coatings of precious metal nanorods to produce a 'smart', spectrally-selective coating. In this scheme the optical properties of the coating are modulated using a concept that we term 'active plasmonic control'. The idea builds on the previous work of Malik Maaza and co-workers ${ }^{7}$ in 2005 , in which the complex optical response of a composite $\mathrm{Au} / \mathrm{VO}_{2}$ nanocoating was examined.

Coatings of nanospheres on transparent substrates are of interest even without active plasmonic control because they can be configured to give spectral selectivity on transmission ${ }^{8,9}$, while angular selectivity is additionally possible with nanorods ${ }^{3}$ or nanocaps ${ }^{10}$. In the case of nanorods this is because the intensity of the longitudinal plasmon resonance also 
depends closely on the polarization and orientation of the incoming light. In principle or in practice these coatings can be configured to block the infrared radiation of sunlight (for windows) ${ }^{11}$, used as dichroic filters ${ }^{3}$, or used as the basis for a color-change device ${ }^{12}$. However, we show here that further functionality can be imparted to the coatings by surrounding the nanoparticles with an active dielectric matrix capable of undergoing an externally stimulated change in optical properties. This is because the position and intensity of a plasmon resonance are also dependent on the dielectric constants of the medium in the immediate vicinity of the nanoparticle. The effect is significantly more pronounced for nanoshells or nanorods than for nanospheres ${ }^{13}$. Electrochromic, thermochromic or other systems based on this phenomenon can be envisaged.

Vanadium dioxide $\left(\mathrm{VO}_{2}\right)$ is a particularly attractive candidate material for the role as 'active dielectric' in a nanoscale coating system. The material undergoes a reversible transition between a semiconductor phase $\left(\mathrm{VO}_{2}-\mathrm{M}\right)$ to a conducting phase $\left(\mathrm{VO}_{2}-\mathrm{R}\right)$ at about $68^{\circ} \mathrm{C}{ }^{14,15}$. In the transition from semiconductor to metal both the electrical conductivity and optical reflectivity of the $\mathrm{VO}_{2}$ change dramatically. The transition is most commonly induced by a change in temperature but is actually electronic in nature. Therefore, it can also be induced isothermally by an injection of photo-carriers or by subjecting the $\mathrm{VO}_{2}$ to a strong electric field ${ }^{15,16}$. The phase change is exceptionally rapid in the latter instances, occurring in less than a picosecond ${ }^{15}$. Several interesting applications of these phenomena in $\mathrm{VO}_{2}$ have been identified, including thermochromic windows, e.g. ${ }^{17}$, optical fiber switching devices ${ }^{16,18}$, holographic storage systems ${ }^{19}$, switchable photonic crystals $^{20}$, and infrared bolometers ${ }^{21}$. Here, however, we will concentrate on showing how the transition in $\mathrm{VO}_{2}$ could be used to modulate a plasmonic response.

\section{CALCULATIONS}

The simulations of the nanorods described here were carried out with the DDSCAT code of Draine and Flatau ${ }^{22,23}$, while those of the spherical core-shell particles used BHCOAT, a code originally from the textbook of Bohren and Huffman that invokes the analytical solution of $\mathrm{Mie}^{24}$. Both programs calculate the extinction, absorption and scattering efficiencies for a nanoparticle, but DDSCAT uses the discrete dipole approximation (DDA), in terms of which the particle is rendered as a 3-D array of dipoles which are then processed numerically. Convergence in materials (such as gold) with high refractive indices ( $n$ and $k$ ) is slow and the dipole volume must be small in order to produce a stable numerical result. In the present work electron scattering off the surfaces has been neglected and bulk dielectric properties have been used. This is considered acceptable if the rods are longer than the mean free path of an electron $(\sim 40 \mathrm{~nm})^{4,25}$, particularly as it is the longitudinal resonance that is of the greatest interest. There are also size-dependent effects on the dielectric constants of $\mathrm{VO}_{2}{ }^{26,27}$ but these are also neglected here and the thin film data of Verleur' ${ }^{27}$ were used 'as is'. In all cases the external environment is taken as vacuum.

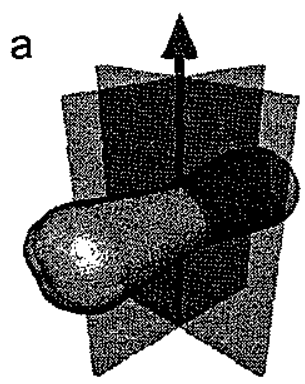

C

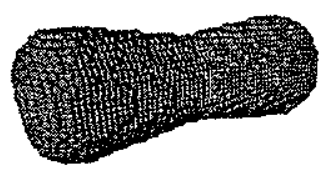

b

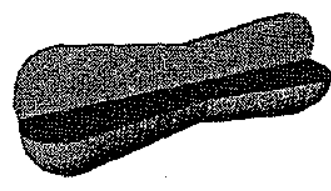

d

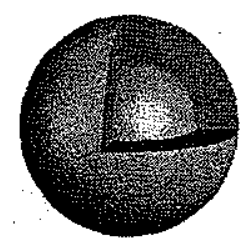

Figure 1. Composite targets investigated in present work consisting of precious-metal core and a $\mathrm{VO}_{2}$ coating, (a) external view of nanorod target, showing polarizations (blue) and propagation vector (red) of the light, (b) cut-away view of nanorod target showing Au core and $\mathrm{VO}_{2}$ shell, (c) nanorod target after rendering into array of dipoles, (d) cut-away

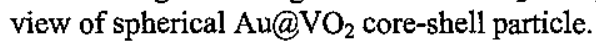


The simulated $\mathrm{VO}_{2}$-coated nanorod target used in the present work is shown in Figure 1(a)-(c). It consists of a gold or silver nanorod core, $80 \mathrm{~nm}$ in length and with a diameter of $20 \mathrm{~nm}$. Vanadium dioxide is then notionally precipitated onto the rod as shown, to form a coating. The composite object was then rendered into 20,000 dipoles, each with a volume of $\sim 2 \mathrm{~nm}^{3}$, for use by the DDSCAT code. Simulations of the optical properties of the Au@VO $\mathrm{VO}_{2}$ core-shell particles, Figure $1(\mathrm{~d})$, and of the solid $\mathrm{VO}_{2}$ spheres were carried out using BHCOAT.

\section{RESULTS}

The simulated optical properties of the composite nanorods are shown in Figure 2, alongside vacuum data for the corresponding naked particle. It is evident that coating the precious-metal nanorods with $\mathrm{VO}_{2}$ is predicted to markedly attenuate their plasmon resonance, and red-shift the extinction peak due to the longitudinal resonance. The position of the latter can be moved back and forth by about $50 \mathrm{~nm}$, simply by causing the reversible phase transformation between $\mathrm{VO}_{2-}$ $\mathrm{M}$ and $\mathrm{VO}_{2}-\mathrm{R}$ to occur. Although attenuated relative to the case of a vacuum environment, the extinction efficiencies of the $\mathrm{VO}_{2}$-coated rods are still large and of technological interest.

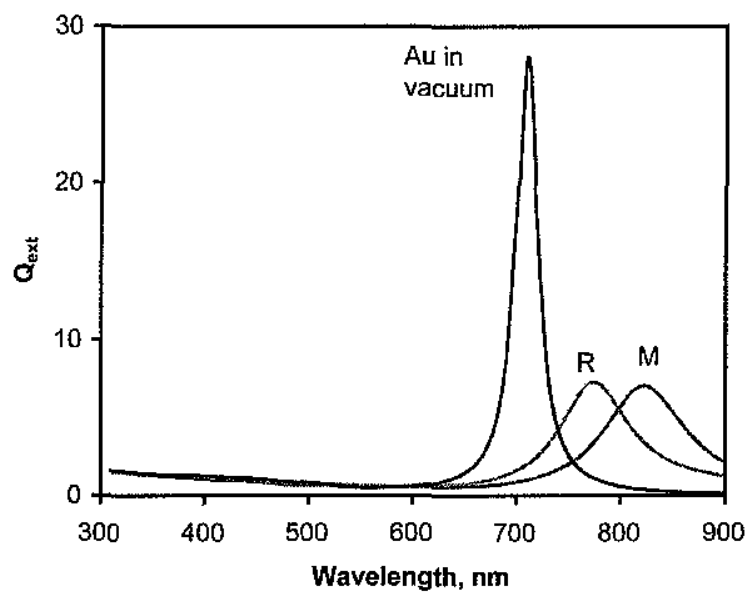

(a)

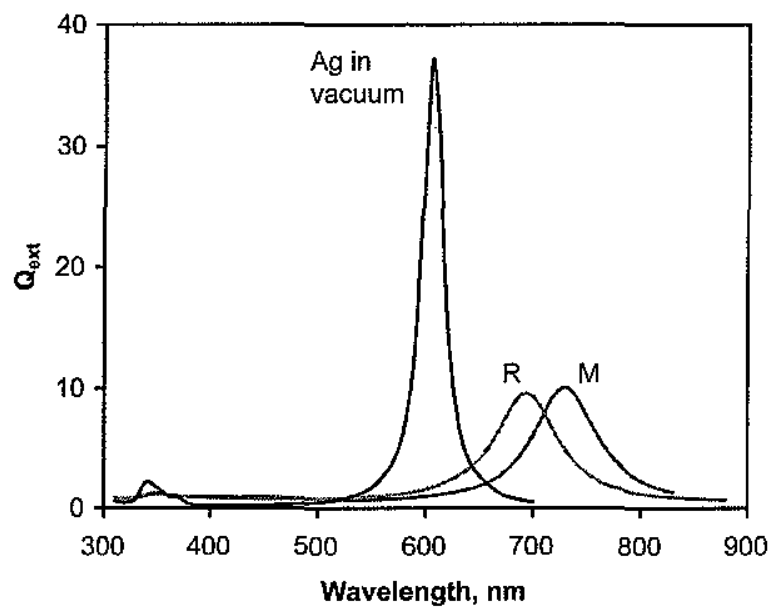

(b)

Figure 2. Modulation of the plasmon response of precious metal nanorods of $4: 1$ aspect ratio that have been coated with $\mathrm{VO}_{2}$ $\mathrm{R}$ is prediction for $\mathrm{VO}_{2}-\mathrm{R}$, the high-temperature metallic phase, $\mathrm{M}$ the prediction for $\mathrm{VO}_{2}-\mathrm{M}$, the low-temperature semiconducting phase. (a) Gold nanorod cores. (b) Silver nanorod cores.

A wavelength-dependent figure-of-merit for the change in optical properties, the 'switching ratio', can be defined:

$$
\phi_{e x t}(\lambda)=\frac{Q_{e x t}^{V O_{2}-M_{1}}(\lambda)}{Q_{e x t}^{V O_{2}-R}(\lambda)}
$$

where $\lambda$ is the wavelength of light at which the figure-of-merit is evaluated.

In practical terms this modulation can be achieved by varying the temperature of a hypothetical coating containing a dispersion of these rods from about 60 to $75^{\circ} \mathrm{C}$ and back again ${ }^{14}$. In addition, an interesting, self-regulating thermal response at particular wavelengths has been predicted ${ }^{13}$ as the result of the thermochromic variation in absorption efficiencies for the two forms of $\mathrm{VO}_{2}$. This would have the effect of 'locking' the temperature of coating onto about $68^{\circ} \mathrm{C}$ provided that the intensity of the illumination fell within a particular range. However, in the present work, the point of greater importance is that there is a wavelength (750 nm for the gold nanorods) at which the particles transmit roughly $2 \frac{1}{2} \mathrm{x}$ more light at high temperatures than at low temperatures, and a second wavelength $(850 \mathrm{~nm})$ in which this relationship is reversed, with the transmission of light at the lower temperature being $2 \frac{1}{2} \mathrm{x}$ greater than at the higher, Figure 3. The corresponding figures for silver are similar, but the wavelengths of light at which the switching in peaks occur are blue shifted to 670 and $750 \mathrm{~nm}$ respectively. Unfortunately, the extinction peaks are not broad or well separated enough to make a meaningful difference for a solar shielding application. However, it is feasible that the effect could be used to modulate a monochromatic beam of appropriate wavelength for some other purpose. It is also 
interesting to note that the semiconductor $\rightarrow$ metal transition can also be very rapidly induced by a laser pulse. In this case, it is not the heat imparted by the laser that causes the transition, rather, the photon-induced change in the electronic structure $^{15}$. This provides additional possibilities for controlling the optical switching ${ }^{16}$.

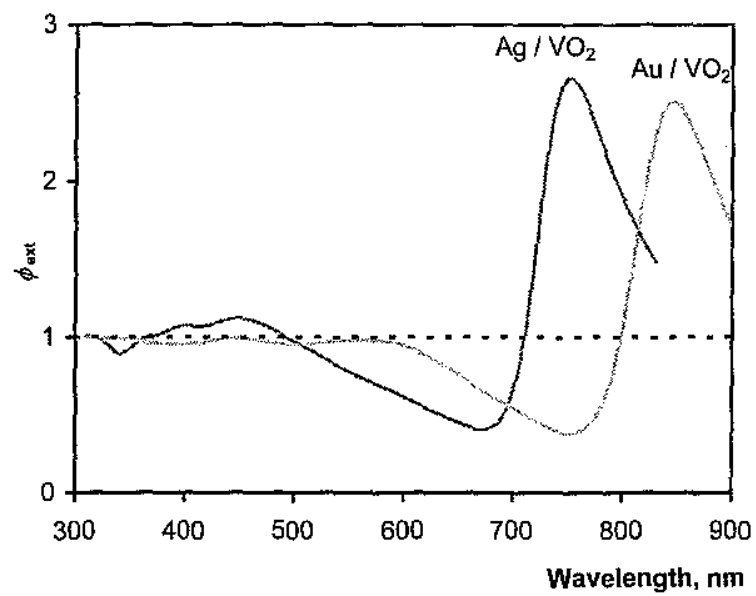

Figure 3. Switching efficiencies of $\mathrm{Ag}$ and $\mathrm{Au}$ nanorods that have been over-coated with $\mathrm{VO}_{2}$. The rods have a 4:1 aspect ratio and are $80 \mathrm{~nm}$ long. Note that the position at which maximum switching occurs can be readily controlled by changing the aspect ratio.

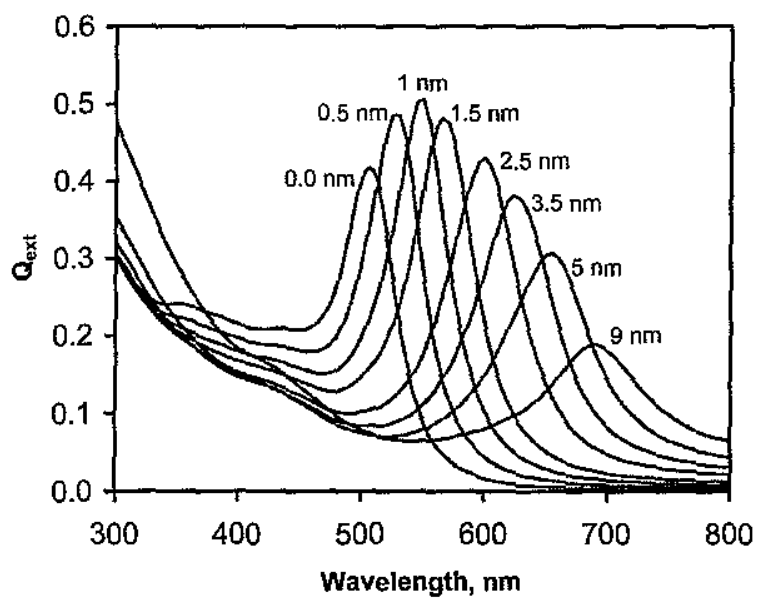

Figure 4. Simulated optical extinction spectra for a series of $\mathrm{Au} @ \mathrm{VO}_{2}$ core-shell particles, with a 13 nm diameter Au core, and the indicated thickness of $\mathrm{VO}_{2}-\mathrm{M}$ shell.

No attempt has been made here to optimize the thickness of the $\mathrm{VO}_{2}$ coating on the nanorods. There are three reasons for this. First, the DDA calculations were computationally intensive, and hence slow, secondly a target with sufficiently fine resolution to contain a thin shell would necessarily need to contain a very large number of dipoles (exacerbating the first factor), and thirdly because, in any case, electron scattering effects in a thin shell would be expected to broaden and attenuate the extinction peaks considerably. On the other hand, some qualitative insight into the probable effect of reducing the thickness of the $\mathrm{VO}_{2}$ layer can be gained by a consideration of the special case of spherical core-shell particles (Figure 1(d)) for which an analytical solution to the optical properties is available. In Figure 4 we show the effect of varying the thickness of a shell of $\mathrm{VO}_{2}-\mathrm{M}$ placed on a Au nanosphere of $13 \mathrm{~nm}$ diameter. For reasons of clarity the corresponding peaks for the $\mathrm{VO}_{2}-\mathrm{R}$ phase are not shown but are in each case blue-shifted and slightly attenuated relative to those for $\mathrm{VO}_{2}-\mathrm{M}$. Switching ratios of nearly $2.5 \mathrm{x}$ are possible in the red part of the visible spectrum for a gold core of $13 \mathrm{~nm}$ diameter. To fully optimize the effect it is also necessary to systematically vary the core diameter as well as shell thickness. If this is done then $\phi_{\text {ext }}$ up to 2.56 seem feasible for Au@ $\mathrm{VO}_{2}$ core-shell spheres ${ }^{13}$. Even higher 
Syxtching Tho that in the near-infrared ${ }^{13}$.

-

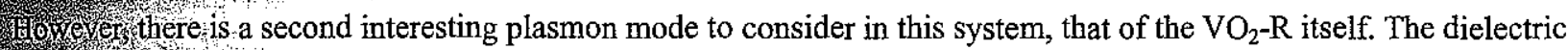
or. V115. cas is somewhat irrelevant. In Figure 5 we show the switchable optical extinction predicted for a $\mathrm{VO}_{2}$ sphere of 100

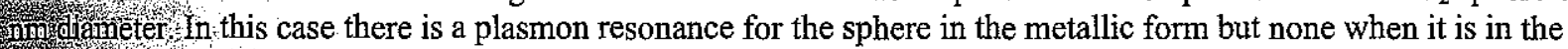
Semeonducting form. Rather than being tunable, here it is a case of being able to turn a plasmon resonance on or off. W. 1. 16 the plasmon resonance of the $\mathrm{VO}_{2}-\mathrm{R}$ sphere peaks at $1030 \mathrm{~nm}$, the maximum extent of switching available is at 전.

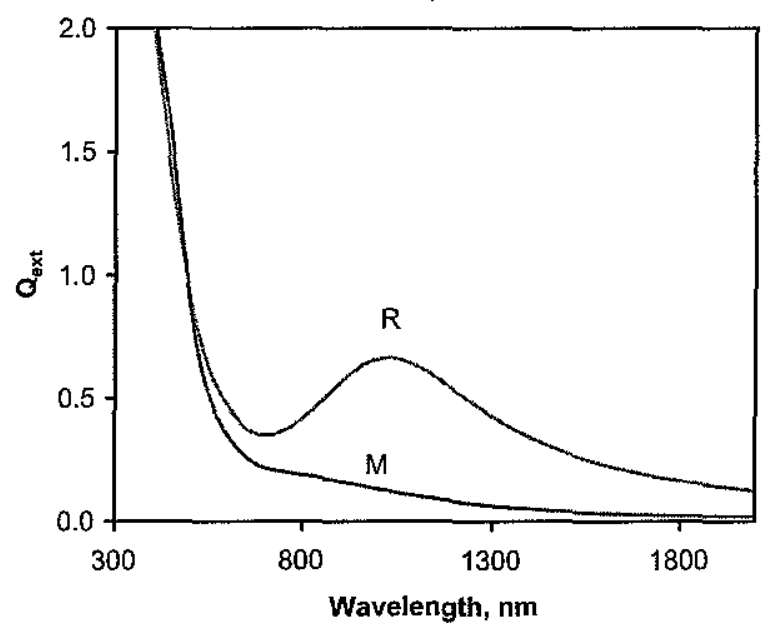

Figure 5. Switchable optical extinction characteristics predicted for a $\mathrm{VO}_{2}$ sphere of $100 \mathrm{~nm}$ diameter. (M and $\mathrm{R}$ as for Figure 2)

Finally, the plasmon resonance of a $\mathrm{VO}_{2}$ shell on an $\mathrm{Au}$ core becomes evident in its own right when the $\mathrm{VO}_{2}$ is present at a sufficiently large thickness. In this case an interesting mixed system results, with a tunable plasmon response available from the $\mathrm{Au}$ core and a switchable one from the $\mathrm{VO}_{2}$ shell, Figure 6.

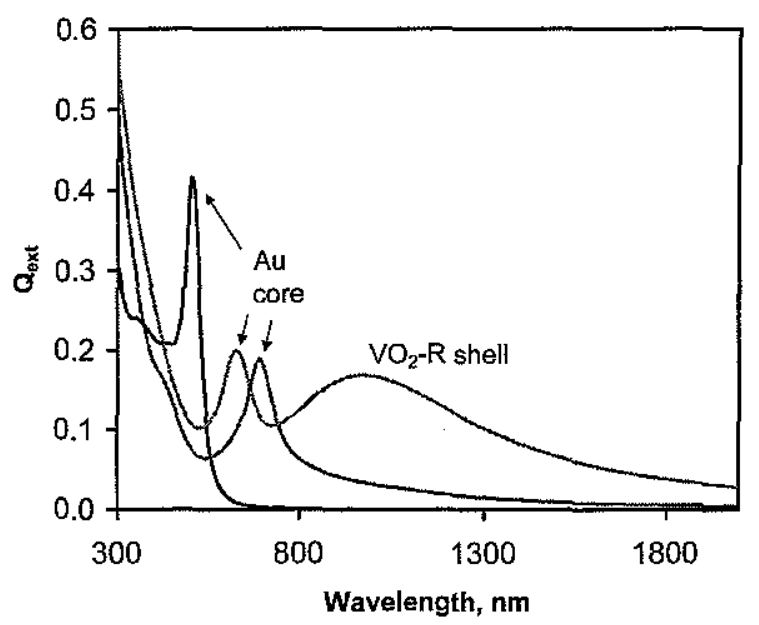

Figure 6. A simulation of the complex, thermochromic plasmonic response available from a $13 \mathrm{~nm}$ diameter Au core that has been over-coated with a $9 \mathrm{~nm}$ thick $\mathrm{VO}_{2}$ shell. There is a tunable plasmon resonance mode due to the core ('core' on the figure) and a switchable resonance due to the shell (' $\mathrm{VO}_{2}-\mathrm{R}$ shell' on the Figure). 


\section{CONCLUSIONS}

The combination of a plasmonic nanostructure with a material with controllable dielectric response offers interesting new functionalities. We have coined the term 'active plasmonic control' to describe the phenomena in these new systems. A plasmon resonance in these systems can be either 'tuned', that is shifted in respect of the wavelength of light at which it occurs, or even switched on or off. Switching ratios of at least $2 \frac{1}{2} \mathrm{x}$ are possible with systems based on coinage metal nanorods or nanospheres that have been over-coated with $\mathrm{VO}_{2}$. In these instances control of the structure of the dielectric phase can be used to 'tune' the wavelength at which a plasmon resonance occurs. However, in the case of $\mathrm{VO}_{2}$ nanospheres the plasmon resonance can actually be switched on or off by control of its structure. A hybrid response, showing both tunablity and switching is expected in some of these composite particles.

\section{REFERENCES}

[1] P. Mulvaney, "Surface plasmon spectroscopy of nanosized metal particles," Langmuir 12, 788-800, 1996.

[2] K. L. Kelly, E. Coronado, L. L. Zhao and G. C. Schatz, "The optical properties of metal nanoparticles: the influence of size, shape, and dielectric environment," J. Phys. Chem. B 107, 668677, 2003.

[3] M. B. Cortie, X. Xu and M. J. Ford, "Effect of composition and packing configuration on the dichroic optical properties of coinage metal nanorods," Phys. Chem. Chem. Phys. 8, 3520-3527, 2006.

[4] K. Ueno, S. Juodkazis, M. Mino, V. Mizeikis and H. Misawa, "Spectral sensitivity of uniform arrays of gold nanorods to dielectric environment," J. Phys. Chem. C 111, 4180-4184, 2007.

[5] X. Huang, I. H. El-Sayed, W. Qian and M. A. El-Sayed, "Cancer cell imaging and photothermal therapy in the near-infrared region by using gold nanorods," J. Am. Chem. Soc. 128, 2115-2120, 2006.

[6] D. Pissuwan, S. M. Valenzuela, M. C. Killingsworth, X. Xu and M. B. Cortie, "Targeted cell destruction using bioconjugated gold nanorods," J. Nanoparticle Res., in press, DOI 10.1007/s11051007-9212-z, 2007.

[7] M. Maaza, O. Nemraoui, C. Sella and A. C. Beye, "Surface plasmon resonance tunability in $\mathrm{Au}-\mathrm{VO}_{2}$ thermochromic nano-composites," Gold Bull. 38, 100-106, 2005.

[8] X. Xu, M. Stevens and M. B. Cortie, "In situ precipitation of gold nanoparticles onto glass for potential architectural applications," Chem. Mater. 16, 2259-2266, 2004.

[9] S. Schelm and G. B. Smith, "Dilute $\mathrm{LaB}_{6}$ nanoparticles in polymer as optimized clear solar control glazing," Appl. Phys. Lett. 82, 4346-4348, 2003.

[10] J. Liu, B. Cankurtaran, L. Wieczorek, M. J. Ford and M. B. Cortie, "Anisotropic optical properties of semi-transparent coatings of gold nano-caps," Adv. Func. Mater. 16, 1457-1461, 2006.

[11] X. Xu, T. Gibbons and M. B. Cortie, "Spectrally-selective gold nanorod coatings for window glass," Gold Bull. 39, 156-165, 2006.

[12] M. B. Cortie, A. McDonagh and P. Mulvaney, "Devices having a variable optical property and processes of making such devices," in Australian Patent Application (2005).

[13] M. B. Cortie, A. Dowd, N. Harris and M. J. Ford, "Core-shell nanoparticles with self-regulating plasmonic functionality," Phys. Rev. B 75, 113405, 2007.

[14] F. J. Morin, "Oxides which show a metal-to-insulator transition at the Néel temperature," Phys, Rev. Lett. 3, 34-36, 1959.

[15] A. Cavalleri, C. Tóth, C. W. Siders, J. A. Squier, F. Ráksi, P. Forget and J. C. Kieffer, "Femtosecond structural dynamics in $\mathrm{VO}_{2}$ during an ultrafast solid-solid phase transition," Phys. Rev. Lett. 87, 237401, 2001. 
[6. M. Soltani, M. Chaker, E. Haddad and R. Kruzelesky, "1 x 2 optical switch devices based on semiconductor-to-metallic phase transition characteristics of $\mathrm{VO}_{2}$ smart coatings " Measurement Science and Technology 17, 1052-1056, 2006.

17] T. D. Manning and I. P. Parkin, "Atmospheric pressure chemical vapour deposition of tungsten doped vanadium(IV) oxide from $\mathrm{VOCl}_{3}$, water and $\mathrm{WCl}_{6}, "$ Journal of Materials Chemistry 14, 2554-2559, 2004.

[18] C. E. Lee, R. A. Atkins, W. N. Giler and H. F. Taylor, "Fiber optic application for thermal switching in vanadium dioxide films," Appl. Optics 28, 4511-4512, 1989.

[19] A. A. Bugayev and M. C. Gupta, "Femtosecond holographic interferometry for studies of semiconductor ablation using vanadium dioxide film," Opt. Lett. 28, 1463-1465, 2003.

[20] D. Xiao, K. W. Kim and J. M. Zavada, "Electrically programmable photonic crystal slab based on the metal-insulator transition in $\mathrm{VO}_{2}, "$ J. Appl. Phys. 97, 106102, 2005.

[21] L. A. L. de Almeida, G. S. Deep, A. M. N. Lima, I. A. Khrebtov, V. G. Malyarov and H. Neff, "Modeling and performance of vanadium-oxide transition edge microbolometers," Appl. Phys. Lett. 85, 3605-3607, 2004.

[22] B. T. Draine and P. J. Flatau, "Discrete-dipole approximation for scattering calculations," J. Opt. Soc. Am. A 11, 1491-1499, 1994.

[23] B. T. Draine and P. J. Flatau, User Guide for the Discrete Dipole Approximation Code DDSCAT 6.1, 2004, http://arxiv.org/abs/astro-ph/0309069, accessed January 2005.

[24] C. F. Bohren and D. R. Huffman. Absorption and Scattering of Light by Small Particles (Wiley, New York, 1998).

[25] S. Link and M. A. El-Sayed, "Shape and size dependence of radiative, non-radiative and photothermal properties of gold nanocrystals," Inter. Rev. Phys. Chem. 19, 409-453, 2000.

[26] A. Gentle, A. I. Maaroof and G. B. Smith, "Nanograin $\mathrm{VO}_{2}$ in the metal phase : a plasmonic system with falling dc resistivity as temperature rises," Nanotechnology 18, 025202, 2007.

[27] H. W. Verleur, A. S. Barker and C. N. Berglund, "Optical properties of $\mathrm{VO}_{2}$ between 0.25 and $5 \mathrm{eV}$," Physical Review 172, 788-798, 1968. 